\title{
Estudios de las propiedades medicinales de extracto total y distintas fracciones de Ligaria cuneifolia (R. et P.) Tiegh (Loranthaceae).
}

\author{
Studies of the medicinal properties of total extract and \\ different fractions of Ligaria cuneifolia (R. et P.) \\ Tiegh (Loranthaceae).
}

\begin{abstract}
Mariana Ferrero ${ }^{1}$, Alicia Dominighini ${ }^{1}$, Leda Urli ${ }^{1}$, Guillermo Mengarelli ${ }^{1}$, Diego Crosetti ${ }^{1}$, José Gonzálvez', Sebastián Galliano', Natasha Gerschcovsky' ${ }^{1}$, Gloria García ${ }^{1}$, Constanza Giacosa $^{1}$, Julián Fisch ${ }^{1}$, María Emilia Pelozzi ${ }^{1}$, Carolina Galiasso ${ }^{1}$, Juan Beloscar ${ }^{3}$, Juan Monti' $^{2}$, María T. Ronco ${ }^{2}$, Daniel Francés ${ }^{2}$, Cecilia Dobrecky ${ }^{4}$, Marcelo Wagner ${ }^{4}$, Cristina Carnovale $^{2}$, Alejandra Luquita ${ }^{1}$.

${ }^{1}$ Cátedra de Biofísica. Facultad de Ciencias Médicas. Universidad Nacional de Rosario. Rosario; Argentina. ${ }^{2}$ Departamento de Ciencias Fisiológicas. Facultad de Ciencias Bioquímicas y Farmacéuticas. Universidad Nacional de Rosario. Instituto de Fisiología Experimental (IFISE)-CONICET. Rosario; Argentina.

${ }^{3}$ Servicio de Cardiología. Hospital Provincial del Centenario. Rosario; Argentina.

${ }^{4}$ Cátedra de Farmacobotánica. Facultad de Farmacia y Bioquímica. Universidad de Buenos Aires. Buenos Aires; Argentina.
\end{abstract}

Autor por correspondencia: Alejandra Luquita - luquitale@hotmail.com Conflicto de intereses: no presenta.

\section{Resumen}

Ligaria cuneifolia (Lc) (R. et P.) Tiegh. (Loranthaceae), popularmente conocida como "muérdago criollo", "liga" o "liguilla", es una especie hemiparásita, que se encuentra en el centro y norte de Argentina. En medicina tradicional es utilizada para disminuir la presión arterial y el colesterol. Basándonos en las observaciones empíricas, planteamos como objetivo demostrar científicamente que Ligaria cuneifolia tiene propiedades hipotensoras e hipocolesterolémicas. En ratas Wistar machos adultas alimentadas con dieta normal, enriquecida en colesterol y en hiperlipémicas (alimentadas con High Fat Diet), demostramos que el tratamiento por vía intraperitoneal con una fracción enriquecida en Proantocianidinas extraídas de Lc (PLc) produce disminución del 40\% del colesterol plasmático (Co) debido al aumento de la velocidad de excreción biliar de sales biliares, así como disminución de triglicéridos y LDLCo, sin producir cambios en la fluidez de la sangre. Evaluamos el efecto vasoactivo de PLc in vitro en anillos de aorta aislada de ratas determinando su tensión isométrica, presentando efecto vasodilatador dependiente de endotelio, ya que la remoción mecánica del mismo anula completamente la relajación. El tratamiento con infusión de extracto liofilizado de hojas y tallos de Lc ingerida tres veces por semana durante dos meses por pacientes

Cita sugerida: Ferrero M, Dominighinil A, Urlil L, Mengarelli1 G, Crosettil D, Gonzálvez J, Galliano S, Gerschcovsky N, García G, Giacosal C, Fisch J, Pelozzi M E, Galiasso C, Beloscar J, Juan Monti J, Ronco M T, Francés D, Dobrecky C, Wagner M, Carnovale C y Luquita A. (2020). Estudios de las propiedades medicinales de extracto total y distintas fracciones de Ligaria cuneifolia (R. et P.) Tiegh (Loranthaceae). Rev Fac Cs Méd UNR. Vol. 1: pp. 72 a 78. 
hipercolesterolémicos de ambos sexos (edad: $50 \pm 15$ años), permitió observar descenso en los niveles Co y LDLCo, sin mostrar alteraciones en la fluidez de la sangre ni en la funcion hepático. Conclusión: Ligaria cuneifolia sería una potencial herramienta en la prevención de enfermedades cardiovasculares, por disminuir el Colesterol plasmático y presentar un marcado efecto hipotensor.

Palabras claves: Ligaria cuneifolia. Proanticianidinas. Hipocolesterolémica. Hipolipémica. Fluidez sanguínea. Hipotensora.

\begin{abstract}
Ligaria cuneifolia (Lc) (R. et P.) Tiegh. (Loranthaceae), popularly known as "Creole mistletoe", "league" or "liguilla", is a hemiparasitic species, found in central and northern Argentina. In traditional medicine it is used to lower both blood pressure and cholesterol. Based on empirical observations, we set the objective of scientifically demonstrating that Ligaria cuneifolia has hypotensive and hypocholesterolemic properties. In adult male Wistar rats fed a normal diet, enriched in cholesterol and hyperlipemic (fed High Fat Diet), we demonstrated that treatment intraperitoneally with a fraction enriched in Proanthocyanidins extracted from Lc (PLc) produces a 40\% decrease in cholesterol plasma (Co) due to an increase in the rate of biliary excretion of bile salts, as well as a decrease in triglycerides and LDLCo, without producing changes in blood fluidity. We evaluated the vasoactive effect of PLc in vitro in isolated rat aortic rings was evaluated by determining its isometric tension, presenting an endothelium-dependent vasodilator effect, since its mechanical removal completely cancels relaxation. Treatment with infusion of lyophilized extract of leaves and stems of Lc ingested three times a week for two months by hypercholesterolemic patients of both sexes (age: $50 \pm 15$ years), allowed to observe decrease in Cho and LDLCho levels, without showing alterations in blood fluidity or liver function. Conclusion: Ligaria cuneifolia is a potential tool in the prevention of cardiovascular diseases, as it reduces plasma cholesterol and has a marked hypotensive effect.

Keywords: Ligaria cuneifolia. Proanticyanidins. Hypocholesterolemic. Hypolipemic. Blood fluidity. Hypotensive.
\end{abstract}

\title{
I. Ligaria cuneifolia (R. et P.) Tiegh. (Loranthaceae)
}

\section{1. Características Taxonómicas}

Ligaria cuneifolia (R. et P.) Tiegh. (Loranthaceae), popularmente conocida con el nombre de "muérdago criollo", "liga” o "liguilla”, pertenece al orden Santalales; este orden es el más importante y ostenta el mayor número de especies dentro de las hemiparásitas. Comprende árboles, arbustos, lianas leñosas o hierbas, siendo la mayoría hemiparásitas de raíces o tallos de otras plantas (1).

A todas las plantas con flores que crecen como parásitos en las ramas de los árboles o arbustos se les llama, en forma generalizada, "muérdagos”. Estas representan, aproximadamente, las tres cuartas partes del total de plantas parásitas (2).

La Ligaria cuneifolia es una especiehemiparásita desprovista deraíces aéreas, que se desarrolla sobre diferentes hospedantes. La etimología de Ligaria procede del término liga, en alusión a la capacidad que tienen sus frutos de "ligar" o atrapar pájaros. En tanto, cuneifolia refiere a la particular forma de cuña de sus hojas. Posee tallos enteros, sin entrenudos, las ramas adultas son cilíndricas y las jóvenes subcomprimidas, ambas rugoso-estriadas. También presenta flores en racimos axilares de color rojo, aunque pueden adquirir coloración anaranjada o a veces amarilla (3). El rango de hospedadores de Lc, a lo largo de su vasta pero discontinua distribución, es muy amplio. De acuerdo con las observaciones de Amico \& Vidal-Russell (2019), se ha encontrado Lc parasitando a más de 30 especies nativas a lo largo de toda su distribución geográfica, con preferencia por leguminosas arborescentes, tales como "chañar" (Geoffroea decorticans (Gill. ex Hook. \& Arn.) Burkart-Fabaceae-), "algarrobo" (Prosopis spp.-Fabaceae-), "churqui” (Acacia caven (Molina) Molina -Fabaceae-), Acacia spp., Robinia spp., "horco-cebil" (Piptadenia excelsa (Griseb) Lillo -Fabaceae-). Además, se la ha encontrado parasitando sobre talas (Celtis iguanaea (Jacq.) Sarg., C. tala Gillies ex Planch. -Cannabaceae-), molles (Schinus spp. -Anacardiaceae-), quebrachos (Schinopsis spp. -Anacardiaceae-) y plantas cultivadas como manzanos, perales, durazneros, granados, olivos, tipas, entre otros $(4,5,6)$.

Lc posee una amplia distribución geográfica desde Perú al centro de la Argentina y sur del Brasil. Habita en tres regiones geográficas: la región de los Andes (a lo largo de la cordillera de los Andes, desde Perú a Argentina y Chile), la región Central (son las sierras centrales de la Argentina: en las provincias de Córdoba y San Luis) y la región Este (el sureste de Sudamérica: Uruguay, Brasil y la Mesopotamia Argentina) (6). 
Tanto en la región Andina como en la Central se encuentra por encima de los 700 m.s.n.m., mientras que en la región Este se encuentra en bajas altitudes. Por lo tanto, Lc se encuentra en una variedad de eco regiones. En la región Andina, está presente en las eco regiones del Bosque seco boliviano, Matorral chileno, Monte y Puna (7). En la región Central, se encuentra solo en el sur de la eco región del Chaco Seco. En la región Este, en el Espinal, Sabana uruguaya y Pampas, donde está asociada a los corredores ribereños (6).

\section{2. Principales constituyentes químicos y compuestos bioactivos}

\section{Compuestos macromoleculares}

Una lectina galactósido-específica presente en el extracto acuoso inhibe la proliferación de las células tumorales LB. La lectina galactósido-específica es el primer componente aislado responsable de una acción biológica en esta especie $(5,8,9)$.

\section{Compuestos aminados}

Los estudios realizados en ejemplares que se desarrollaban sobre diferentes hospedantes, y recogidos en distintas áreas geográficas, demostraron la presencia de tiramina (10). La concentración detectada en la mayoría de los ejemplares no sobrepasaba los $10 \mathrm{mg} \%$, si bien se detectaron ejemplares con niveles superiores de 100 mg\%. En los individuos que parasitan Geoffroea decorticans (H. et Arn.) Burkart (Fabaceae), se detectaron los niveles más altos, con valores que van de 120 a $360 \mathrm{mg}$ de tiramina por $100 \mathrm{~g}$ de material vegetal seco.

\section{Compuestos terpénicos}

Fueron aislados en esta especie dos compuestos mayoritarios caracterizados como triterpenos pentacíclicos del grupo del lupeol: betulina y ácido betulínico (11).

\section{Flavonoides}

Se caracterizó el flavonol quercetina libre y glicosidado: quercetina-3-O-glucósido, quercetina-3-Oxilósido, quercetina-3-O-arabinopiranósido, quercetina-3-O-arabinofuranósido, quercetina-3-Oramnósido. Además, 4 compuestos galoil derivados: quercetina-3-O-a-L- (3"'-O-galoil) ramnósido, quercetina-3-O-a-L- (2"-O-galoil) ramnósido, quercetina-3-O-a-D-(2"galoil)-arabinofuranósido y quercetina-3-O-a-L-(2"'-O-galoil)-arabinopiranósido (11, 12, 13, 14, 15). Por otro lado, se detectaron, en poblaciones de Lc que crecen en Perú y norte de la Argentina, kaempferol libre y glicosidado; y naringenina (1,13). Además, se detectaron leucoantocianidinas, catequina-4-ß-oly proantocianidinas en distinto grado de polimerización: polímeros, oligómeros y dímeros, que, por tratamiento ácido, producen cianidina $(11,12,16,17)$.

En general la droga vegetal cruda está constituida por las hojas y los tallos jóvenes herbáceos de Ligaria cuneifolia. El extracto etanólico contiene como mínimo $21,0 \pm 0,2 \%$ de fenoles totales (calculado como mg ácido gálico/100 mg extracto), $14 \pm 0,2 \%$ de taninos totales (calculado como mg ácido gálico/100 mg extracto), 0,47 \pm 0,02 de taninos condensados (calculado como Absorbancia/mg extracto), $1,5 \pm 0,1 \%$ de flavonoides (calculado como mg rutina/100 mg extracto) y $1,2 \pm 0,1 \%$ de ácidos hidroxicinámicos (calculado como mg ácido clorogénico/100 mg extracto) (15).

\section{3. Uso en Medicina tradicional}

En “Contribuciones a la Materia Médica” de Juan A. Domínguez (18) aparecen los primeros estudios farmacológicos y fitoquímicos sobre los muérdagos argentinos. Estos estudios se realizaron con ejemplares de Psittacanthus cuneifoliius (Ruiz et Pav.) Engl. (sinonimia de L. cuneifolia) y Phrygilanthus flagellaris (Cham. et Schltdl.) Eicher (sinonimia de Tripodanthus flagellaris; Cham. et Schltdl.) Tiegh. y se observó que la acción hipotensora se verificaba en ejemplares que parasitaban especies de Prosopis mientras que aquellos recogidos sobre Aspidosperma actuaban como adrenérgicos hipertensores.

En su compilación sobre “700 hierbas medicinales” de Argentina, Burgstaller (19) menciona para 
"liga" o para muérdago su acción hipotensora e incluye además su utilización en arteriosclerosis, saturnismo, menopausia, glaucoma y para la esterilidad.

Por otro lado, se presenta la posibilidad de utilizar las hojas, los tallos tiernos y las flores en cocimiento al 20-25\%, atribuyéndole propiedades diuréticas y tónico-cardíacas, pero sobre todo hipotensora. También se muestra que el zumo de muérdago criollo, preparado como extracto acuoso de hojas y ramas jóvenes (100 g en 600 g de agua) tiene propiedades para emplearse como hipotensor y anti-escleroso (34 gotas de zumo corresponden a $1 \mathrm{~g}$ del vegetal) (20).

\section{Aportes realizados por nuestro grupo de investigación al área del conocimiento}

Basándonos en las observaciones empíricas descriptas en I.3., nos planteamos como objetivo de nuestros trabajos demostrar en modelos animales (II.1) y estudios clínicos (II.2) que esta especie botánica tiene propiedades hipotensoras e hipocolesterolémicas.

\section{1. Estudios de tratamiento de extracto total de Lc y fracciones enriquecidas en distintos flavonoides en atas Wistar.}

II. 1.a Evaluación del efecto sobre niveles plasmáticos de colesterol y triglicéridos, la función hepática y renal, y parámetros hemorreológicos

En estudios biológicos realizados en nuestro laboratorio, demostramos que el tratamiento de ratas normocolesterolémicas por vía intraperitoneal (ip) con el extracto crudo de Lc en dosis crecientes produce un aumento de la viscosidad sanguínea, como consecuencia de un aumento en la rigidez eritrocitaria. Esta rigidización de la membrana del eritrocito podría deberse, en parte, a la modificación de la relación fosfolípido-colesterol de esta. En este sentido, el colesterol (Co) de la membrana eritrocitaria se intercambia en forma dinámica con el Co plasmático; por ende, es altamente infuenciado por sus modificaciones. En nuestros estudios, observamos que el tratamiento con el extracto crudo de Lc condujo a una disminución del 40 \% del Co plasmático. Esta disminución en el nivel de Co se debe a un incremento en la velocidad de excreción biliar de Co (VEBSB) y de las sales biliares (productos de la metabolización hepática del Co). El aumento en la excreción biliar de estos componentes conduce a un aumento del flujo biliar total (FB) (21).

Tabla 1: Efecto de las diferentes dosis de Lc sobre los parámetros hemorreológicos.

\begin{tabular}{|l|c|c|c|c|c|c|c|}
\hline \multicolumn{7}{|c|}{ Dosis crecientes de Lc en mg/100 g PC } \\
\hline & Control & $\mathbf{1 , 5}$ & $\mathbf{2 , 5}$ & $\mathbf{3 , 5}$ & $\mathbf{5 , 5}$ & $\mathbf{8 , 3}$ & $\mathbf{1 7}$ \\
\hline $\begin{array}{l}\text { A VSrs } \\
\text { (centipoise) }\end{array}$ & $4,65 \pm 0,43$ & $6,31 \pm 0,25$ & $8,47 \pm 0,75^{* *}$ & $7,36 \pm 0,20^{*}$ & $7,27 \pm 0,35^{*}$ & $6,15 \pm 0,35^{*}$ & $4,52 \pm 0,69$ \\
\hline B IR & $8,73 \pm 0,62$ & $7,20 \pm 0,57$ & $8,47 \pm 0,75^{* *}$ & $13,67 \pm 1,98^{*}$ & $13,00 \pm 0,99^{*}$ & $10,35 \pm 1,08^{*}$ & $14,5 \pm 1,71^{*}$ \\
\hline C $\Delta$ Colesterol & - & $19,66 \pm 3,90^{*}$ & $17,25 \pm 1,65^{*}$ & $15,00 \pm 3,51^{*}$ & $18,60 \pm 3,00^{* *}$ & $17,35 \pm 0,29^{*}$ & $19,8 \pm 6,07^{*}$ \\
\hline
\end{tabular}

A: Viscosidad sanguínea relativa estandarizada a un hematocrito del 45\%: VSrs= (Vs/Vp)45/ Hto, medida con viscosímetro Brookfield LVT-PC a una velocidad de corte de 230 s-1. B: Índice de rigidez eritrocitaria (IR) determinado mediante filtración con membrana de nucleopor de una suspensión diluida (buffer fosfato-albúmina) de eritrocitos (hematocrito 10\%). C: La disminución en el nivel de colesterol plasmático se calculó utilizando la ecuación: $\Delta \mathrm{Col}(\mathrm{mg} / \mathrm{ml}$ ) = Col basal-Col post-Lc. Colbasal: Concentración plasmática de colesterol determinada en sangre, antes de la inyección del extracto de Lc. ColpostLc: Concentración plasmática de colesterol luego de la inyección de extracto. Los valores representan el promedio de seis animales \pm ES. *Diferencia significativa con respecto al grupo control $(\mathrm{p}<0,05) .{ }^{* *}$ Diferencia significativa con respecto al grupo control $(\mathrm{p}<0,01$ 
Tabla 2: Efecto de las diferentes dosis de Lc sobre el flujo y parámetros biliares.

\begin{tabular}{|c|c|c|c|c|c|c|c|}
\hline \multicolumn{7}{|c|}{ Dosis crecientes de Lc en mg/100 g PC } \\
\hline & Control & $\mathbf{1 , 5}$ & $\mathbf{2 , 5}$ & $\mathbf{3 , 5}$ & $\mathbf{5 , 5}$ & $\mathbf{8 , 3}$ & $\mathbf{1 7}$ \\
\hline A & $1,59 \pm 0,20$ & $1,31 \pm 0,18$ & $2,28 \pm 0,19^{* *}$ & $1,99 \pm 0,10^{*}$ & $1,76 \pm 0,14^{*}$ & $2,15 \pm 0,2^{*}$ & $1,99 \pm 0,23$ \\
\hline B & $42,70 \pm 3,0$ & $49,4 \pm 4,0$ & $83,1 \pm 5,0^{* *}$ & $73,8 \pm 2,8^{*}$ & $75,5 \pm 4,7^{*}$ & $64,2 \pm 5,01^{*}$ & $51,44 \pm 5,78$ \\
\hline C & $0,79 \pm 0,08$ & $1,02 \pm 0,15$ & $1,34 \pm 0,19^{*}$ & $1,57 \pm 0,03^{* *}$ & $0,95 \pm 0,18$ & $1,43 \pm 0,18^{*}$ & $0,79 \pm 0,08$ \\
\hline
\end{tabular}

A: Flujo biliar expresado en $\mu \mathrm{l} / \mathrm{min}$.g de hígado. B: Excreción de sales biliares. C: Excreción de colesterol biliar expresada en nmol/ min.g de hígado. Los valores representan el promedio de seis animales \pm ES. * Diferencia significativa con respecto al grupo control $(\mathrm{p}<0,05) .{ }^{* *}$ Diferencia significativa con respecto al grupo control $(\mathrm{p}<0,01)$.

Posteriormente, en estudios realizados en nuestro laboratorio, demostramos que el tratamiento de ratas por vía ip con la fracción metanólica de Lc (FMLc) causa una disminución de la deformabilidad eritrocitaria, evidenciado por una interacción de la FMLc con la membrana eritrocitaria. El cambio de forma del eritrocito de discocito a estomatocito y el aumento de la rigidez eritrocitaria producen el aumento de la viscosidad sanguínea observada. Asimismo, observamos un descenso de Co plasmático, como consecuencia del aumento de la excreción biliar de Co y de las sales biliares y por consiguiente, del flujo biliar (22).

Prosiguiendo con el análisis de las distintas fracciones extraídas de Lc, realizamos estudios in vivo e in vitro con la fracción enriquecida en quercetina (QLc) y la fracción enriquecida en catequina (catLc). A diferencia de lo demostrado con la administración del extracto crudo de Lc, por vía ip, con QLc y catLc, no evidenciamos cambios significativos en los parámetros de excreción biliar. Tampoco registramos un descenso en los niveles plasmáticos de Co. En los estudios in vitro, observamos que la incubación de sangre, extraída de las ratas por punción cardíaca, con distintas concentraciones de QLc 2,3 mg/100 g PC o 0,60 mg/100 g por PC catLc durante 3 días, produce un cambio en la forma del glóbulo rojo de discocito a estomatocito y, al mismo tiempo, origina una rigidización eritrocitaria (menor deformabilidad), conduciendo a un aumento de la viscosidad sanguínea. Estos cambios podrían deberse a la interacción de QLc o de catLc con la membrana eritrocitaria y a una disminución del volumen eritrocitario. Estos resultados sugieren que otro componente de Lc sería el responsable del descenso de Co plasmático observado (23).

Posteriormente, realizamos el estudio con otra fracción extraída de Lc, enriquecida con el flavonoide proantocianidina (PLc). El tratamiento de ratas normocolesterolémicas por vía ip produjo un descenso significativo en los niveles plasmáticos de Co concomitante con un aumento de la VEBSB y de sales biliares, sin producir cambios en la viscosidad sanguínea, es decir, sin alterar la fluidez de la sangre (24).

Recientemente, el tratamiento en ratas alimentadas con una dieta enriquecida en Co, con diferentes dosis de PLc administradas durante 3, 7 y 10 días por vía ip, indujo una disminución de los niveles de Co plasmático debido al aumento de la VEBSB y del FB. La disminución del Co plasmático podría deberse al incremento de la VEBSB, lo cual, por el aumento de entes osmóticamente activos, explicaría el aumento del FB (25).

Con el objetivo de analizar el efecto de una dosis de $3 \mathrm{mg} / 100 \mathrm{~g}$ de peso corporal de PLc sobre los niveles plasmáticos de Co, desarrollamos un modelo de ratas hiperlipémicas, utilizando una dieta rica en grasa (del inglés High Fat Diet - HFD) preparada con dieta estándar adicionada con $40 \%$ de primer jugo bovino (cada 100g: 1,2g de Co, 1,06g de grasa total y 6,8g de proteínas), por un período de 28 días. A los 28 días de ingesta de la dieta, les extrajimos sangre de la cola para determinar los valores basales de hematocrito, Co, CoHDL y CoLDL. Una vez confirmado el aumento de Co, comenzamos a inyectar por vía ip a los animales cada 24 horas, 3, 7 y 10 días consecutivos, con solución fisiológica al grupo Control y con solución del extracto enriquecido en Proantocianidinas (PLc) al grupo Tratadas. Al respecto, en ratas hiperlipémicas (HFD), el tratamiento con PLc durante 3, 7 y 10 días consecutivos, disminuyó el Co total y CoLDL, así como los TG. La disminución de Co puede ser debido al incremento de la VEBSB, que conduciría al aumento del FB. También, pudimos observar que no produce alteraciones en las enzimas hepáticas ni cambios en la viscosidad sanguínea ni en la forma eritrocitaria, a saber, no altera la fluidez de la sangre (26).

Los resultados nos permiten considerar a la PLc como una potencial herramienta en la prevención 
de la enfermedad cardiovascular, al reducir el CoLDL plasmático, uno de los principales factores de riesgo en el desarrollo de aterosclerosis y que contribuye al desarrollo de cardiopatías.

\section{II.1.b Evaluación del efectodeproantocianidina extraída de Lc sobre sobrelacontractibilidad de aorta aislada de rata.}

Para analizar el efecto hipotensor del extracto enriquecido en PLc, se realizaron estudios in vitro del potencial efecto vasoactivo sobreanillos deaorta aislada de ratas. Se disecóla aorta torácica dividiéndose en anillos, éstos se colocaron en una cámara y la tensión isométrica de los vasos se midió usando un transductor de desplazamiento de fuerza conectado a un polígrafo. Posteriormente, los anillos fueron contraídos con una solución despolarizante de Krebs-Henseleit modificada, procedimiento que se repitió hasta conseguir una contracción máxima estable. Luego de 10 min de estabilización, el vaso fue contraído con fenilefrina (Phe) $(0,1 \mathrm{mM}$ ) y, una vez que alcanzó una contracción máxima estable, se agregó una fracción enriquecida en PLc en concentraciones crecientes $\left(7,14 \times 10^{-6} ; 7,14 \times 10^{-5} ; 7,14 \times 10^{-4}\right.$; $7,14 \times 10^{-3} ; 7,14 \times 10^{-2} ; 7,14 \times 10^{-1} ;$ y $71,4 \mathrm{mg} / \mathrm{mL}$ ), con el propósito de construir una curva concentraciónrespuesta. En la Figura 1 puede observarse los resultados obtenidos.

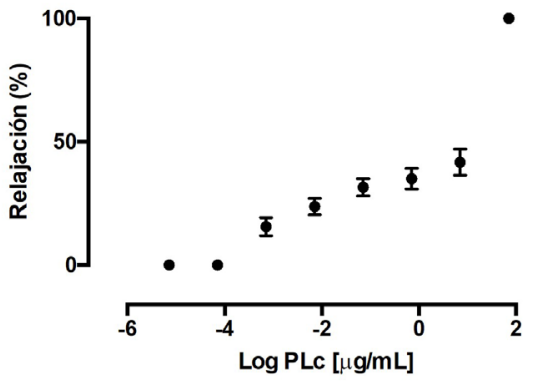

Figura 1: Curva concentración-respuesta de PLc en anillos aislado de aorta de rata.

La concentración de PLc, que indujo la relajación máxima, fue de 71,4 mg/ml. Utilizando una ecuación logística de 4 parámetros $(\mathrm{R} 2=0.92)$, estimamos que la concentración del sustrato, que produce un $50 \%$ de relajación (EC50), es de $6,86 \mathrm{mg} / \mathrm{mL}(\mathrm{n}=4)$. PLc tiene efecto vasodilator, que resulta dependiente de endotelio, ya que la remoción mecánica del mismo anula completamente la actividad relajadora de PLc (27).

\section{2. Análisis del efecto de infusiones de extracto total Lc en pacientes.}

Se estudiaron pacientes de ambos sexos (edad: $50 \pm 15$ ) años con antecedentes de hipercolesterolemia. Todos los pacientes firmaron el consentimiento informado correspondiente y una copia de este fue archivado. Luego, recibieron sobres con extracto liofilizado de hojas y tallos de Lc (2,6 gr c/u), para preparar infusión con agua caliente, para ser ingerida tres veces por semana durante dos meses. Se realizaron determinaciones en sangre en el tiempo 0 y a los 60 días: Colesterol total (Co), HDL y LDL, Triglicéridos, Viscosidad Sanguínea Relativa, Forma Eritrocitaria, determinándose el Índice Morfológico (IM) y hepatograma. En los pacientes estudiados, Lc generó un descenso del colesterol plasmático total y LDL, sin modificar HDL ni Triglicéridos, sin promover alteraciones en la forma de los eritrocitos ni en viscosidad sanguínea. Por otro lado, no observamos cambios en los parámetros evaluados de la función hepática. Es decir, que en pacientes que recibieron infusión de Ligaria cuneifolia observamos descenso en los niveles plasmáticos de colesterol total y LDL, sin mostrar alteraciones en la fluidez de la sangre ni en la función hepática. $(27,28)$.

\section{Conclusión}

Los resultados obtenidos nos permiten concluir que especialmente la fracción enriquecida en Polifenoles obtenida de Ligaria cuneifolia es una potencial herramienta en la prevención de la enfermedad cardiovascular, disminuyendo el Co plasmático, que es uno de los principales factores de riesgo en el desarrollo de ateroesclerosisy que contribuye al desarrollo de cardiopatías. Además, presenta un marcado efecto hipotensor que sería un efecto coadyuvante de gran importancia como cardioprotector. 
Agradecimientos: Facultad de Ciencias Médicas, Consejo de Investigaciones de la Universidad Nacional de Rosario (Argentina) e Instituto de Fisiología Experimental (CONICET, Argentina).

Fuentes de financiamiento: Facultad de Ciencias Médicas, Consejo de Investigaciones de la Universidad Nacional de Rosario (Argentina) e Instituto de Fisiología Experimental (CONICET, Argentina).

\section{Referencias Bibliográficas}

1. Takhtajan A. Diversity and classification of flowering plants. New York: Columbia University Press; 1997, p.11-19.

2. Feuer S, Kuijt J. Fine structure of mistletoe pollen. Eremolepidaceae, Lepidoceras and Tupeia. Can J Bot. 1978; 56:2853-2864.

3. Subils, R. Loranthaceae. Los géneros de Fanerogamas de la Argentina. In: Hunziker AT Editor. Bol. Soc. Arg. Bot. 1984;24 (1-2): 176.

4. Abbiatti D. Las Lorantáceas Argentinas. Rev Mus La Plata (N. S.), Sec Bot. 1946; 7:1-110.

5. Varela B, Fernández T, Taira C, et al. El muérdago criollo, Ligaria cuneifolia (R. et P.) Tiegh. -Loranthaceae-. Desde el uso popular hacia el estudio de los efectos farmacológicos. Dominguezia. 2001;17(1):31-50.

6. Amico G, Vidal-Russell R. Uso de hospedadores por el muérdago argentino Ligaria cuneifolia (Loranthaceae) a lo largo de su distribución geográfica. In: Hunziker AT Editor. Bol. Soc. Arg. Bot. 2019; 54:395-403.

7. Olson D, Dinerstein E, Wikramanayake E, et al. Terrestrial Ecorregions of the World: A New Map of Life on Earth: A new global map of terrestrial ecoregions provides an innovative tool for conserving biodiversity. Biosci.2001; 51:933-938.

8. Fernández T, Cerdá Zolezzi P, Aulicino P, et al. Immunobiological features of the galactoside lectin L-Lc isolated from the Argentine mistletoe Ligaria cuneifolia. J Ethnopharmacol 2003; 85(1):81-92.

9. Cerdá Zolezzi P, Fernández T, Aulicino P, et al. Ligaria cuneifolia flavonoid fractions modulate cell growth of normal lymphocytes and tumor cells as well as multidrug resistant cells. Immunobiol. 2005; 209:737-749.

10. Vázquez y Novo S, Wagner M, Gurni A, et al. Importancia Toxicológica de la presencia de sustancias aminadas en ejemplares de L. cuneifolia var. cuneifolia colectados en distintas áreas de la República Argentina. Acta farm. bonaer. 1989;8(1):23-29.

11. Wagner ML, Fernández T, Varela B, et al. Anatomical, phytochemical and immunochemical studies on Ligaria cuneifolia (R. et P.) Tiegh. (Loranthaceae). Pharm. Biology. 1998; 36(2):131-139.

12. Fernández T, Wagner M, Varela B, et al. Study of an Argentine Mistletoe, the hemiparasite Ligaria cuneifolia (R. et P.) Tiegh. (Loranthaceae). J Ethnopharmacol. 1998; 62:25-34.

13. Arenas Chávez C, Vera Gonzáles C, Valeriano Zapana J, et al. Identificación de polifenoles individuales y evaluación de la actividad anticancerígena de la Ligaria cuneifolia (Liga-liga). Revista ECIPERÚ. 2011; 8(2):158-162. ISSN: 1813 - 0194

14. Soberón J, Sgariglia M, Dip Maderuelo M, et al. Antibacterial activities of Ligaria cuneifolia and Jodina rhombifolia leaf extracts against phytopathogenic and clinical bacteria. J Biosci Bioeng. 2014; 118(5):599-605.

15. Dobrecky C, Moreno E, Garcés M, et al. Contenido de polifenoles en Ligaria cuneifolia y su relación con la capacidad antioxidante. Dominguezia. 2014;30(2):35-39

16. Dobrecky C, Flor S, López P, et al. Development of a novel dual CD-MEKC system for the systematic flavonoid fingerprinting of Ligaria cuneifolia (R. et P.) Tiegh -Loranthaceae- extracts. Electrophoresis. 2017; 38:1292-1300.

17. Wagner ML, Fernández T, Álvarez E, et al. Comparación de Componentes Micro y Macromoleculares del Muérdago Criollo (Ligaria cuneifolia (R. et P.) Tiegh.) y del Muérdago Europeo (Viscum album L.). Acta farm. bonaer. 1996; 15 (2): 99-108.

18. Domínguez JA. Loranthaceae. In: Contribuciones a la Materia Médica Argentina. Trabajos del Instituto de Botánica y Farmacología. 1928;44: 260-264.

19. Burgstaller J. 700 hierbas medicinales. Ed. Lancelot. Buenos Aires, Argentina. 2000; 224.

20. González Torres D. Catálogo de Plantas medicinales (y alimenticias útiles) usadas en Paraguay. Editorial Sevilibro Asunción, Paraguay. 2018;456

21. Mengarelli G., Dominighini A, Ferraro M, et al. Evaluation of hemorheologic parameters and biliary secretion in Ligaria cuneifolia (Argentine mistltoe) extracted treated rats. Pharm Biol. 2001;39(6): 435-439.

22. Ferrero M., Crosetti D., Dominighini A, et al. Effect of the vehicle polyvinylpyrrolidone and the Methanolic Fraction of Ligaria cuneifolia (Argentine Mistletoe) extract on hemorheological properties and biliary secretion in rats. Clin Hemorheol Microcirc. 2007; 36(2): 95-104.

23. Dominighini A, Ferrero M, Crosetti D, et al. Effect of Ligaria cuneifolia catechin- and quercetin- enriched fractions on hemorheology and plasma cholesterol. Clin Hemorheol Microcirc. 2010; 44: 1-9.

24. Dominighini A, Ferrero M, Crosetti D, et al. Effects of proanthocyanidin enriched extract from Ligaria cuneifolia on plasma cholesterol and hemorheological parameters.In vivo and In vitro studies. Clin Hemorheol Microcirc. 2015; 60: 317-325.

25. Gonzálvez J, García G, Galliano S, et al. The enriched proanthocyanidin extract of Ligaria cuneifolia shows a marked hypocholesterolemic effect in rats fed with cholesterol-enriched diet. Recent Pat Endocr Metab Immune Drug Discov. 2018;11(1):47-53.

26. Gerschcovsky N, Knox M, Carnovale C, et al. Efecto biológico de proantocianidina extraída de Ligaria cuneifolia sobre la contractibilidad de aorta aislada de rata. Medicinal plant communications.2018;38: http://solaplamed.org/wp-content/uploads/2019/01/MPC-1-1-4-2018-23-39.pdf.

27. Ferrero M, Crosetti D, Svetaz M, et al. Evaluación de la actividad hipocolesterolémica de infusiones de Ligaria cuneifolia en pacientes con riesgo cardiovascular Dominguezia 2013; 29(S): 102-103.

28. Ferrero M, Svetaz MJ, Giacosa C, et al. Efecto de la infusión de Ligaria cuneifolia o Muérdago criollo sobre el perfil lipídico en pacientes con hipercolesterolemia. Argentine Cardiology J. 2015; 44(1): 25 